\title{
Lipoprotein (a) and Atherogenic Index of Plasma are Indicators for Endothelial Dysfunction in Pregnancy-induced Hypertensive Women
}

\author{
Visala Sree Jammalamadaga ${ }^{1}$, Palem Siva Prasad ${ }^{2}$
}

\begin{abstract}
Introduction: $\mathrm{PIH}$ is one of the most common causes of both maternal and neonatal morbidity. Endothelial dysfunction (ED) is a classic hallmark for PE. The mechanisms involved in the induction of ED are poorly understood. The disorders in lipoprotein metabolism are one of the most important causes for ED. AIP is another risk factor calculated by log (triglyceride/HDL-c) which is an indicator of atherosclerosis. The role of $\mathrm{Lp}(\mathrm{a})$ in atherosclerosis and its ability to induce thrombosis makes it important in normal and complicated pregnancies. So, a study has been designed to analyse the levels of Lp(a), Atherogenic index of plasma (AIP), and Nitric oxide (NO) in PIH women.

Materials \& Methods: A cross-sectional analytical study involved normotensive, PE \& E pregnant women with 100 subjects in each group. They were investigated for serum Lp(a), NO in the 3rd trimester of pregnancy. Lp(a) was estimated by commercial kit method, NO was measured by Griess method and AIP was calculated by standard formula log (triglyceride/HDL-c).

Results: The systolic blood pressure (SBP), diastolic blood pressure (DBP), AIP were significantly high and NO levels were significantly low between 3 groups. The Lp(a) levels were significantly high in PE than controls. The Lp(a) and AIP were negatively correlated with NO.

Conclusion: $\mathrm{LP}(\mathrm{a})$ and AIP are indicators to predict endothelial dysfunction in preeclampsia and eclampsia subjects. AIP may be better predicable indicator than $\mathrm{LP}(\mathrm{a})$, since it was significantly raised in both $\mathrm{PE}$ and $\mathrm{E}$.
\end{abstract}

Keywords: Atherogenic Index of Plasma, Eclampsia, Endothelial Dysfunction, Lipoprotein (a), Preeclampsia.

Indian Journal of Medical Biochemistry (2019): 10.5005/jp-journals-10054-0100

\section{INTRODUCTION}

Pregnancy induced hypertension (PIH) is one of the most common cause of both maternal and neonatal morbidity. Worldwide, $10 \%$ of all pregnancy women's are complicated by hypertension, with preeclampsia (PE) and eclampsia (E) being the major causes of maternal and prenatal morbidity and mortality. ${ }^{1}$

In developing countries all over the world, about $8-10 \%$ of pregnant women's suffer with preeclampsia. ${ }^{2}$ The research findings solidly confirm that the maternal mortality rate (MMR) is 400 out of 100,000 live births. In Africa 1:16 life time risk is recorded which is the highest, compared to the Western nations (1:2800). Eclampsia accounts for $12 \%$ of such deaths. ${ }^{3}$ Approximately $12-25 \%$ of fetal growth restrictions, small for gestational age babies as well as $15-20 \%$ of all preterm births are attributable to preeclampsia. ${ }^{4}$ In developing countries, the preeclampsia and eclampsia are leading threats to safe motherhood where women's are seven times more likely to develop $\mathrm{PIH}$. In this scenario, it is assessed that $10-25 \%$ of cases (an estimated 40000 women) lead to maternal deaths annually. ${ }^{5}$ The national incidence of PIH is $15.2 \%$ in India, while it is four times higher in primipara women than in multipara. ${ }^{6}$

Pregnancy induced hypertension is the appearance of hypertension of more than 140/90 $\mathrm{mm}$ of $\mathrm{Hg}$ after 20 weeks of gestation. When hypertension is allied with significant proteinuria it is called preeclampsia. Pre-eclampsia complicated by seizures is called eclampsia which is a major cause of maternal mortality. ${ }^{7}$

Endothelial dysfunction (ED) plays a focal role in the origin of multisystem disorder that develops in preeclampsia. The mechanisms involved in the induction of endothelial dysfunction are poorly understood. Pregnancy is associated with physiological
${ }_{1,2}$ Assistant Professor

${ }^{1}$ Department of Biochemistry, Sambhram Institute of Medical Sciences, KGF, Kolar, Karnataka, India

${ }^{2}$ Department of Biochemistry, Basaveshwara Medical College and Hospital, Chitradurga, Karnataka, India

Corresponding Author: Palem Siva Prasad, Assistant Professor, Department of Biochemistry, Basaveshwara Medical College and Hospital, Chitradurga, Karnataka, India, e-mail: sp.biocom@yahoo. co.in

How to cite this article: Jammalamadaga VS, Prasad PS. Lipoprotein (a) and Atherogenic Index of Plasma are Indicators for Endothelial Dysfunction in Pregnancy-induced Hypertensive Women. Indian J Med Biochem 2019;23(2):263-266.

Source of support: Nil

Conflict of interest: None

changes such as increase in lipids from first to third trimester in order to satisfy energy and membrane demands of the developing fetus. But, in pregnancy related disorders such as PE, maternal plasma lipid levels were abnormally exaggerated. ${ }^{8}$ The disorders in lipoprotein metabolism are one of the most important causes for ED. Endothelial dysfunction is an early index of atherogenesis. ${ }^{9}$ Atherogenic index of plasma (AIP) has been recently proposed as marker of plasma atherogenecity in coronary heart disease. Atherogenic index of plasma is calculated by standard formula Log (triglyceride/HDL-C). ${ }^{10}$

Lipoprotein-(a) $[\mathrm{Lp}(\mathrm{a})]$ is a subclass of lipoprotein, consisting of a low-density lipoprotein (LDL) covalently bound via its apolipoprotein-B100 portion to apolipoprotein-(a). ${ }^{11}$ It is a

() The Author(s). 2019 Open Access This article is distributed under the terms of the Creative Commons Attribution 4.0 International License (https://creativecommons. org/licenses/by-nc/4.0/), which permits unrestricted use, distribution, and non-commercial reproduction in any medium, provided you give appropriate credit to the original author(s) and the source, provide a link to the Creative Commons license, and indicate if changes were made. The Creative Commons Public Domain Dedication waiver (http://creativecommons.org/publicdomain/zero/1.0/) applies to the data made available in this article, unless otherwise stated. 
neglected element of the blood lipid profile. It is recognized as a determinant of coronary heart disease progression and its role in atherosclerosis and its ability to induce thrombosis make it potentially important in the course of normal and complicated pregnancies. ${ }^{12}$ So, a study has been designed to analyse the levels of $\mathrm{Lp}(\mathrm{a})$, nitric oxide (NO) and atherogenic index in pregnancy induced hypertensive women.

\section{Materials and Methods}

A cross sectional analytical study was conducted in the inpatient ward of the Department of Obstetrics and Gynecology, Annapoorana institute of medical sciences \& hospital (AMC\&H), Salem, Tamil Nadu from August 2012 to April 2017. The study was approved by the Institutional Ethics Committee of AMC\&H and informed consent was obtained from all participants. PIH patients were defined according to the NHBPEP (National high blood pressure education programme) guidelines. ${ }^{13}$

\section{Patients and Controls}

Total 300 subjects were selected for the study. Among these subjects 100 were normotensive pregnant woman who served as control (Group 1), 100 were pre-eclamptic women (Group 2) and remaining 100 are eclamptic women (Group 3). We separated the study groups based on data collected from patient file at hospital as follows. The mean systolic blood pressure (SBP) in 3 groups was recorded as ( $116 \pm 5.45$ vs. $162.18 \pm 18.26$ vs. $170 \pm 15.52) \mathrm{mm} \mathrm{Hg}$. The mean diastolic blood pressure (DBP) in 3 groups was recorded as (75 \pm 5.99 vs. $107.5 \pm 11.35$ vs. $112.28 \pm 10.59) \mathrm{mm} \mathrm{Hg}$. Pre-eclampsia was defined as having a systolic blood pressure $140 \mathrm{~mm} \mathrm{Hg}$ or a diastolic blood pressure $90 \mathrm{~mm} \mathrm{Hg}$ with proteinuria $300 \mathrm{mg} / \mathrm{dL}$. Signs and symptoms of PIH like swelling in the hands, face and feet, severe headaches, abdominal pain, reduced output of urine or no urine, blood in the urine, a change in reflexes, convulsion/ seizures, coma, dizziness, excessive vomiting, nausea and rapid gain in weight were recorded. Eclamptic complications like cerebro vascular, cardiovascular, visual, pulmonary, renal, hepatic, hemostatic and obstetrical were also recorded.

Exclusion criteria: Individuals with past history of cardiac, renal, hepatic illness, diabetes, and hypertensions were excluded.

Sampling: Five milliliters of ante-cubital venous blood sample was collected from each subject after overnight fasting. Plane tubes were used for serum and sodium citrate tube was used for plasma. Plasma and serum were separated by centrifugation at $3000 \mathrm{rpm}$ for 20 minutes.

Serum Lp(a) was estimated using immuno-turbidimetric method ${ }^{14}$ commercially available standard kit (Agappe diagnostics) in a semiautoanalyser. Triglyceride and $\mathrm{HCL}-\mathrm{C}$ were measured by Glycerol phosphate oxidase and peroxidase and ImmunoInhibition, 2 Reagent methods. ${ }^{15,16}$ The Atherogenic index of plasma was calculated by using a formula log (TG / HDL-C). Nitric oxide (NO) was done by using Griess method. ${ }^{17}$

Statistical analysis: The data was processed on computer software package SPSS version 20. The numerical data was presented as Mean \pm SD. One way ANOVA was used to evaluate mean differences in maternal serum Lp(a), NO and AIP concentrations between patients and controls. A value of $\mathrm{P}<0.05$ at $95 \% \mathrm{Cl}$ was considered as statistically significant. Correlation was done by using pearson correlation analysis.

\section{Results}

Table 1 shows the socio demographic characters between Controls, $\mathrm{PE}$ and $\mathrm{E}$ groups. The Maternal age, Gestational weeks were almost comparable between the 3 groups. The mean SBP/DBP was significantly high in preeclampsia, eclampsia groups compared to controls. The eclamptic women had displayed significantly higher SBP\& DBP levels compared to the pre-eclamptic women.

Table 2 shows the levels of Lp (a), Atherogenic index of plasma (AIP) and NO between Controls, PE and E groups. The levels of Lp (a), AIP are significantly high and NO levels were significantly low in both PE \& E women compared to normotensive pregnant women. The $L p(a)$ levels were non-significantly high, AIP was significantly high, NO levels were significantly low in eclamptic women compared to the pre-eclamptic women.

Table 3 shows the correlation analysis between Lp(a) and AIP

Table 1: Sociodemographic characters between controls, PE and E groups

\begin{tabular}{|c|c|c|c|c|c|c|c|c|c|}
\hline \multirow[b]{2}{*}{ Parameters } & \multicolumn{2}{|c|}{ Controls } & \multicolumn{2}{|c|}{ Preeclampsia } & \multirow[b]{2}{*}{ 'P'value } & \multicolumn{2}{|c|}{ Eclampsia } & \multirow[b]{2}{*}{ 'P'value } & \multirow{2}{*}{$\begin{array}{l}\text { 'P' value } \\
\text { between PE \&E }\end{array}$} \\
\hline & Mean & $S D$ & Mean & $S D$ & & Mean & $S D$ & & \\
\hline Age (Years) & 23.97 & 3.30 & 24.62 & 4.07 & NS & 25.71 & 3.71 & $<0.01^{* *}$ & NS \\
\hline Gestational age (weeks) & 31.57 & 2.67 & 32.42 & 3.12 & NS & 31.05 & 2.90 & NS & NS \\
\hline $\mathrm{SBP}(\mathrm{mm} \mathrm{Hg})$ & 116 & 5.45 & 162.18 & 18.26 & $<0.01$ & 170 & 15.52 & $<0.01^{* *}$ & $<0.01^{* *}$ \\
\hline $\mathrm{DBP}(\mathrm{mm} \mathrm{Hg})$ & 75 & 5.99 & 107.5 & 11.35 & $<0.01$ & 112.28 & 10.59 & $<0.05^{*}$ & $<0.01^{* *}$ \\
\hline
\end{tabular}

*Data expressed as mean \pm SD. A p value of $<0.05$ is considered as significant.

${ }^{* *}$ Data expressed as mean \pm SD. A p value of $<0.01$ is considered as highly significant.

Table 2: Lp (a), atherogenic index of plasma (AIP) and NO between study groups.

\begin{tabular}{|c|c|c|c|c|c|c|c|c|c|}
\hline \multirow[b]{2}{*}{ Parameters } & \multicolumn{2}{|c|}{ Controls } & \multicolumn{2}{|c|}{ Pre-eclampsia } & \multirow[b]{2}{*}{ 'p'value } & \multicolumn{2}{|c|}{ Eclampsia } & \multirow[b]{2}{*}{ 'p'value } & \multirow{2}{*}{$\begin{array}{l}\text { 'P'value between } \\
P E \text { and } E\end{array}$} \\
\hline & Mean & $S D$ & Mean & $S D$ & & Mean & $S D$ & & \\
\hline $\operatorname{Lp}(\mathrm{a})(\mathrm{mg} / \mathrm{dl})$ & 48.86 & 11.21 & 72.92 & 13.44 & $<0.01^{*}$ & 75.76 & 11.63 & $<0.01^{*}$ & NS \\
\hline $\mathrm{NO}(\mu \mathrm{mol} / \mathrm{L})$ & 117.37 & 14.77 & 43.8 & 6.13 & $<0.01^{*}$ & 38.6 & 9.94 & $<0.01^{*}$ & $<0.01^{*}$ \\
\hline AIP & 0.30 & 0.09 & 0.48 & 0.08 & $<0.01^{*}$ & 0.56 & 0.12 & $<0.01^{*}$ & $<0.01^{*}$ \\
\hline
\end{tabular}

*Data expressed as mean \pm SD. A p value of $<0.01$ is considered as highly significant. 
Lp(a) and AIP are Risk Factors to cause Endothelial Dysfunction in PIH Women

Table 3: Correlation analysis of NO with between Lp(a) and AIP

\begin{tabular}{lll}
\hline & \multicolumn{2}{c}{ Nitric oxide } \\
\cline { 2 - 3 } Parameter & 'r'value & ' $p^{\prime}$ 'value \\
\hline Lp(a) & -0.689 & $0.000^{*}$ \\
AIP & -0.687 & $0.000^{*}$ \\
\hline
\end{tabular}

*Pearson's Correlation among NO, Lp(a) and AIP

with endothelial dysfunction marker (NO). The Lp(a) and AIP were negatively correlated with Nitric oxide.

\section{Discussion}

Pregnancy induced hypertension, being the prominent cause of maternal death and perinatal morbidity, the necessity for a dependable marker in order to identify the disease in early stages is increasing gradually. Endothelial dysfunction is considered as a center to the multiple-organ pathophysiology of preeclampsia-eclampsia. ${ }^{18}$ The clinical feature of PE is new onset of maternal hypertension and proteinuria that resolves after delivery. The release of seizure-provoking factors or other damaging proteins or pro-inflammatory cytokines like TNF-a, HsCRP, IL-6 due to maternal endothelial dysfunction in preeclampsia may lead to Blood brain barrier disruption and increased permeability which may cause seizure onset. ${ }^{19}$ The impairment in the bioavailability of NO is considered as hallmark of endothelial dysfunction. ${ }^{20} \mathrm{NO}$ is an endothelium derived factor required for vasodilatation and platelet activation inhibition. It is also involved in different stages of pregnancy including implantation, maintenance of uterine acquiescence during pregnancy, control of uterine contractions and relaxation, basic physiological adaption for fruitful gestation and regulation of blood pressure. ${ }^{21}$ Our analysis had shown a significantly lower NO levels in PE \& E women compared to the normotensive women. Eclamptic women had displayed significantly lower NO levels than pre-eclamptic indicating that the endothelial dysfunction plays an important role in the progression and complication of the disease. ED also observed in several other conditions like diabetes, smoking and physical inactivity. ${ }^{22}$ One of the factor for ED is hyperlipidemia. ${ }^{23}$

In pregnancy, lipolysis of TG-rich lipoproteins like chylomicrons, VLDL is reduced because of decreased lipolytic activities of the mother, whereas placental VLDL receptors are up-regulated. ${ }^{24}$ This results in a rerouting of TG-rich lipoproteins to the fetoplacental unit. However, in PIH, the vascularization of the fetoplacental unit may be impaired, resulting in yet-undefined compensatory mechanisms that may further increase synthesis of maternal TG levels. In addition, the decreased catabolism of TG -rich lipoproteins results in the accumulation of TG- rich remnant lipoproteins in the maternal circulation. Remnant lipoproteins may induce platelet activation and endothelial dysfunction, thus leading to the major clinical symptoms of $\mathrm{PIH}^{25}$ The similarity between this and the process of atherosclerosis together with the antifibrinolytic properties of $L p(a)$ has led to consider the role of $L p(a)$ in preeclampsia.

Lipoprotein-(a) is a subclass of lipoprotein, consisting of a low-density lipoprotein (LDL) covalently bound via its apolipoprotein-B100 portion to apolipoprotein-(a) [Apo(a)]. $\mathrm{Lp}(\mathrm{a})$ forms an important part of the clinical biomarker profile of patients with CHD and heart failure. In addition to the role of $L p(a)$ in atherosclerosis, the Apo(a) element of $L p(a)$ has a structure similar to plasminogen, allowing $L p(a)$ particles to reduce the physiological fibrinolytic activity of plasminogen by competitively binding endothelial plasminogen receptors. It is this pro-thrombotic effect, together with $\operatorname{Lp}(a)$ accumulation in atherosclerotic lesions that is thought to predispose those with high levels to $\mathrm{CHD} .{ }^{26}$ In the current study the Lp(a) levels were significantly high in pre-eclamptic and eclamptic women compared to the normotensive pregnant women. The $L p(a)$ levels were non-significantly high in eclamptic women compared to the PE women. In agreement with our study, few studies had demonstrated that the higher $L p(a)$ levels were associated with severity of disease. ${ }^{27-29}$ This increase might be due to more widespread endothelial cell damage, necessitating increased levels of $L p(a)$ to act both as an acute phase protein and as a vehicle for cholesterol deposition at the site of dysfunction. ${ }^{30}$ However some studies had demonstrated no change in the level of $L p(a)$, this might be due to the differences in the method used, study design, sample sizes and ethnicity of study population. ${ }^{31,32}$ It was also stated that the association between Lp(a) and cardiovascular outcomes may differ by race/ethnicity. ${ }^{33}$

In the present study the atherogenic index of plasma (AIP) was calculated. The mean of AIP in PE and E was significantly higher than that of control group. In eclamptic women the AIP was significantly higher than PE women. The AIP was calculated as log (TG/HDL-C) has been proposed as a marker of plasma atherogenicity. ${ }^{34}$ Our findings were in agreement with other studies. ${ }^{35} \mathrm{~A}$ positive significant correlation between AIP and systolic blood pressure was observed by Singh, $\mathrm{M}$ et al., $2015 .^{36}$ This might be due to elevated TC level, reduced $\mathrm{HDL}-\mathrm{C}$ level and $\mathrm{ROS}$ which may lead to the fatty deposition in the vessel walls. This may predispose the patients for coronary heart disease in future. Our results can be interpreted in such a way that plasma lipids in normal pregnancy are at atherogenic levels and were more abnormal in preeclampsia and eclampsia. These abnormalities in lipid profile turn out to be a risk factor for cardiovascular complications. Evaluation of the atherogenic indices during pregnancy may help to prevent this risk.

Endothelial dysfunction was significantly negative correlation with AIP and $L p(a)$. These parameters are significant factors which develop pathological changes in PIH and leads to vascular complications. Since, the present study has proved the correlation among AIP, Lp(a) \& endothelial dysfunction. The elevated levels of Lp(a) \& AIP may be considered as better indicators to predict endothelial dysfunction and its complications in $\mathrm{PIH}$.

\section{ConCLUSION}

Our results indicate that the women with PE \& E exhibit markedly elevated concentrations of TGL-rich lipoproteins. The negative correlation of NO with AIP and Lp(a) indicates the high risk of endothelial dysfunction in PIH women.

So, early careful monitoring of $L p(a)$ and AIP might be helpful to predict the commencement and advancement of ED. It may help in emerging approaches for diagnosis and prevention of maternal and fetal complications.

\section{Limitations OF THE StUdY}

1. The placental histopathological studies could not be done.

2. Further study needs to be focus on parity of patients. 


\section{References}

1. Maas AHEM, Van't Hof AWJ, De Boer MJ. Cardiovascular risk in women after metabolic complications in pregnancy. Netherlands Heart J 2007;15(12): 415-417.

2. Asghari, E., Faramarzi, M., \& Mohammmadi, A. K. The Effect of Cognitive Behavioural Therapy on Anxiety, Depression and Stress in Women with Preeclampsia. Journal of Clinical and Diagnostic Research: JCDR 2016; 10(11): QC04.

3. Shaheen, A., Khan, R. N., Fatima, S., Ali, R., Khan, I., \& Khattak, S. Adipokine Serum visfatin level in pregnancy induced hypertension and uncomplicated pregnancy. Pak J Med Sci 2016; 32(6): 1419-1424.

4. Jeyabalan, A. Epidemiology of preeclampsia: impact of obesity. Nutrition reviews 2013; 71(suppl 1): S18-S25.

5. Agrawal, S., \& Fledder Johann, J. Hypertensive disorders of pregnancy and risk of diabetes in Indian women: a cross-sectional study. BMJ open 2016; 6(8): e011000.

6. Saxena, S., Srivastava, P. C., Thimmaraju, K. V., Mallick, A. K., Dalmia, K., \& Das, B. Socio-demographic profile of pregnancy induced hypertension in a tertiary care centre. Sch J App Med Sci 2014; 2(6D): 3081-3086.

7. Kintiraki E, Papakatsika S, Kotronis G, Goulis DG, Kotsis V. Pregnancyinduced hypertension: review. Hormones 2015;14(2):211-223.

8. Hubel CA. Oxidative stress in the pathogenesis of preeclampsia. Proc Soc Exp Biol Med 1999;222(3):222-235

9. Eleni Anastasiou, MD, John P Lekakis, MD, Maria Alevizaki, MD, PHD, Christos M Papamichael, MD, John Megas, MD, Athanasios Souvatzoglou, MD et al. Impaired endothelium-dependent vasodilatation in women with previous gestational diabetes. Diabetes Care 1998; 21(12): 2111-2115.

10. Meenakshi S, Saikia pathak M \& Paul A. A study on atherogenic indices of pregnancy induced hypertension patients as compared to normal pregnant women. JCDR 2015; 9(7): BC05-BC08.

11. Dube JB, Boffa MB, Hegele RA, Koschinsky ML. Lipoprotein(a): more interesting than ever after 50 years. Curr Opin Lipidol 2012;23: 133-40.

12. Rey E, Kahn SR, David M, Shrier I. Thrombophilic disorders and fetal loss: a meta-analysis. Lancet 2003; 361:901-908.

13. Gifford R.W. Report of the national high blood pressure education program working group on high blood pressure in pregnancy. Am J Obstet Gynecol 2000;183(1):S1-S5.

14. Isser, H. S., Puri, V. K., Narain, V. S., Saran, R. K., Dwivedi, S. K., \& Singh, S. (2000). Lipoprotein (a) and lipid levels in young patients with myocardial infarction and their first- degree relatives. Indian heart Journal 2001; 53(4): 463-466.

15. Oboh FO, Enobhayisobo El. Effect of aqueous extract of Vernonia amygdalina leaves on plasma lipids of hyperlipidaemia adult male albino New Zealand rabbits. Afri Sci. 2009;10(4).

16. Grove TH. Effect of reagent $\mathrm{pH}$ on determination of highdensity lipoprotein cholesterol by precipitation with sodium phosphotungstate-magnesium. Clinical Chemistry. 1979 Apr 1;25(4):560-564.

17. Sun J, Zhang $X$, Broderick $M$, Fein H. Measurement of nitric oxide production in biological systems by using griess reaction assay. Sensors. 2003 Aug 22;3(8):276-284.

18. Schwartz RB. Preeclampsia-Eclampsia: Clinical and Neuroradiographic correlates and insights into the pathogenesis of hypertensive encephalopathy. Radiology 2000; 217: 371-376.

19. Johnson, Abbie Chapman, "Mechanisms of Seizure during Pregnancy and Preeclampsia". graduate college dissertations and theses 2015; Paper 336.
20. Cristina M. Sena, Ana M. Pereira, Raquel Seiça. Endothelial dysfunction - A major mediator of diabetic vascular disease. Biochimica et Biophysica Acta 2013; 1832 (12): 2216-2231.

21. Tanmay Saha, et al. Role of nitric oxide, angiogenic growth factors and biochemical analysis in preeclampsia. Indian journal of biochemistry and biophysics 2013; 50: 462-466.

22. Peramaiyan Rajendran, Thamaraiselvan Rengarajan, Jayakumar Thangavel, Yutaka Nishigaki, Dhanapal Sakthisekaran, Gautam Sethi, et al. The Vascular Endothelium and Human Diseases. Int J BiolSci 2013; 9(10):1057-1069.

23. Sameer Ansar, Juraj Koska1 and Peter D Reaven. Postprandial hyperlipidemia, endothelial dysfunction and cardiovascular risk: focus on incretins. Cardiovascular Diabetology 2011;10:61

24. Wittmaack, F M, Gåfvels, M E, Bronner, M, Matsuo, H, McCrae, K R Tomaszewski, J E., ...\& Strauss 3rd, J F Localization and regulation of the human very low density lipoprotein/apolipoprotein-E receptor: trophoblast expression predicts a role for the receptor in placental lipid transport. Endocrinology 1995; 136(1): 340-348.

25. Winkler, K, Wetzka, B, Hoffmann, M M, Friedrich, I, Kinner, M, Baumstark, M W, ...\& März, W Triglyceride-rich lipoproteins are associated with hypertension in preeclampsia. The Journal of Clinical Endocrinology \& Metabolism 2003; 88(3): 1162- 1166.

26. Angela E Fanshawe (MA, MBBS), Michael Ibrahim (MA, PhD), The current status of lipoprotein (a) in pregnancy: A literature review. Journal of Cardiology 2013; 61: 99-106.

27. Wang, Y, Gu, Y, Zhang, Y, \& Lewis, D F Evidence of endothelial dysfunction in preeclampsia: decreased endothelial nitric oxide synthase expression is associated with increased cell permeability in endothelial cells from preeclampsia. American journal of obstetrics and gynecology 2004; 190(3): 817-824.

28. Bayhan, G, Koçyigit, Y, Atamer, A, Atamer, Y, \& Akkus, Z Potential atherogenic roles of lipids, lipoprotein (a) and lipid peroxidation in preeclampsia. Gynecological endocrinology 2005; 21(1): 1.

29. Nazli, R, Khan, M A, Akhtar, T, Lutfullah, G, Mohammad, N S, Ahmad, J \& Aslam, H Abnormal Lipid levels as a risk factor of eclampsia, study conducted in tertiary care Hospitals of Khyber Pakhtunkhwa ProvincePakistan. Pakistan journal of medical sciences 2013; 29(6): 1410.

30. Fanshawe, A E, \& Ibrahim, M The current status of lipoprotein (a) in pregnancy: A literature review. Journal of cardiology 2013;61(2):99-106.

31. Cesur, M, Ozbalkan, Z, Temel, M A, \& Karaarslan, Y Ethnicity may be a reason for lipid changes and high $\mathrm{Lp}$ (a) levels in rheumatoid arthritis. Clinical rheumatology 2007; 26(3): 355-361.

32. Işildak, M Y, Yildirmak, S T, Doğan, S, Çakmak, M, \& Özakin, E Serum Homocysteine and Lipoprotein (a) Levels in Preeclamptic Pregnants. Balkan Medical Journal 2009; 26(3): 208-213.

33. Banerjee, D, Wong, E C, Shin, J, Fortmann, S P, \& Palaniappan, L Racial and ethnic variation in lipoprotein (a) levels among Asian Indian and Chinese patients. Journal of lipids, 2011.

34. Dobiasova, M AlP--atherogenic index of plasma as a significant predictor of cardiovascular risk: from research to practice. Vnitrni lekarstvi 2006; 52(1): 64-71.

35. Aragon-Charris, J, Reyna-Villasmil, E, Guerra-Velasquez, M, MejiaMontilla, J, Torres- Cepeda, D, Santos-Bolívar, J, \& Reyna-Villasmil, N Atherogenic index of plasma in patients with preeclampsia and in healthy pregnant women. Medicina clinica 2014; 143(3): 104-108.

36. Singh, M, Pathak, M S, \& Paul, A A study on atherogenic indices of pregnancy induced hypertension patients as compared to normal pregnant women. Journal of clinical and diagnostic research JCDR 2015; 9(7): BC05 